Title page

\title{
Title
}

\section{Is there a relationship between blood group and COVID-19 susceptibility?}

\author{
Authors: Davide Golinelli ${ }^{1}$, Erik Boetto ${ }^{2}$, Maria Pia Fantini ${ }^{1,2}$ \\ ${ }^{1}$ Department of Biomedical and Neuromotor Sciences (DIBINEM), Alma Mater Studiorum - University of \\ Bologna. Via San Giacomo 12, 00126, Bologna, Italy. \\ ${ }^{2}$ School of Hygiene and Preventive Medicine, Alma Mater Studiorum - University of Bologna. Via San \\ Giacomo 12, 00126 Bologna, Italy.
}

\section{Authors' Affiliations:}

Davide Golinelli, MD: Department of Biomedical and Neuromotor Sciences (DIBINEM), Alma Mater Studiorum - University of Bologna. Via San Giacomo 12, 00126, Bologna, Italy.

E-mail: davide.golinelli@unibo.it

Erik Boetto, MD: School of Hygiene and Preventive Medicine, Alma Mater Studiorum - University of Bologna. Via San Giacomo 12, 00126 Bologna, Italy.

Email: erik.boetto@gmail.com

Maria Pia Fantini, MD: Department of Biomedical and Neuromotor Sciences (DIBINEM), Alma Mater Studiorum - University of Bologna. Via San Giacomo 12, 00126, Bologna, Italy.

E-mail: mariapia.fantini@unibo.it

\section{Corresponding author:}

Davide Golinelli, MD: Department of Biomedical and Neuromotor Sciences (DIBINEM), Alma Mater Studiorum - University of Bologna. Via San Giacomo 12, 00126, Bologna, Italy.

E-mail: davide.golinelli@unibo.it

\begin{abstract}
Objective. On March 11, 2020 the WHO declared that COVID-19 is pandemic. Among the risk factors for many infectious diseases, a role of the ABO blood group system is reported in the literature. We argue whether it is necessary to investigate the relationship between $A B O$ blood groups and susceptibility to SARS-CoV-2 infection and if we should consider some blood groups as potential risk factors for COVID-19.

Results. Based on the scientific evidence reported in this letter, we believe that further studies are needed to investigate how the ABO polymorphism influences the host susceptibility, individual response and clinical risk for SARS-CoV-2 infection.
\end{abstract}

\section{Keywords}

ABO blood groups system; COVID-19; susceptibility; SARS-CoV-2; SARS 


\section{Introduction}

The outbreak of novel Coronavirus (SARS-CoV-2) officially started on December 31, 2019 in Wuhan, Hubei Province (China), and rapidly spread all over the world, due to the globalization and speed of people movements. On March 11, 2020 the World Health Organization declared that the Coronavirus disease-2019 (COVID-19) is pandemic [1].

In order to fight such a global threat, the scientific community quickly activated and the first studies on the new pathogen's features were conducted and published [2]. Since then, patients' characteristics, clinical presentation and risk factors of SARS-CoV-2 have been extensively analyzed [3].

Starting from the first studies by Helmbold and Vogel in the early '60s [4], among the risk factors for many infectious diseases, a role of the ABO blood group system is reported in the literature. To date, several studies on Coronaviridae viruses (e.g. SARS-CoV [5]) and other viral families like Retroviridae (e.g. HIV [6]) or Hepadnaviridae (e.g. HBV [7]) investigated the host susceptibility linked to the blood group or anti-histo-blood group antibodies.

We argue whether it is necessary to deepen the study of the relationship between ABO blood groups and susceptibility to SARS-CoV-2 infection and if we should consider some blood groups as potential risk factors for COVID-19. We sought in the literature attempting to find existing evidence answering the following question: is there a relationship between blood group and COVID-19 susceptibility?

\section{Main text}

At the time of writing, the only two studies investigating the relationship between blood group and SARS-CoV-2 infection are those of Jiao Zhao et al. [8] and Chen et al. [9] The latter, however, utilizes a subsample of the population already included in Zhao meta-analysis, and provides concordant results.

Jiao Zhao et al. study investigates the relationship between the $A B O$ blood group system and the COVID-19 susceptibility. The Authors compared the ABO blood group distribution in 2,173 patients with COVID-19 confirmed by SARS-CoV-2 test from three hospitals in the Wuhan and Shenzen regions (China) with that in normal people from the corresponding regions. The results showed that blood group distribution remarkably differed from that of the control group in the corresponding region. For example, the ABO blood group in 3,694 normal people in Wuhan displayed a percentage distribution of $32.16 \%, 24.90 \%, 9.10 \%$ and $33.84 \%$ for $A, B, A B$ and $O$, respectively, while the 1,775 patients with COVID-19 from Wuhan Jinyintan Hospital showed an $\mathrm{ABO}$ distribution of $37.75 \%, 26.42 \%, 10.03 \%$ and $25.80 \%$ for $\mathrm{A}, \mathrm{B}, \mathrm{AB}$ and $\mathrm{O}$, respectively. Taking into account the pooled data from the three hospitals by random effects models, blood group $A$ was associated with a higher risk for acquiring COVID-19 (OR, 1.21; 95\% Cl 1.02 1.43, P=0.027) compared with non-A blood groups, whereas blood group $\mathrm{O}$ was associated with a lower risk for the infection (OR, 0.67; 95\% Cl 0.60 0.75, P<0.001) compared with non-O blood groups.

Zhao et al. findings are congruous with other studies that investigated the association between $\mathrm{ABO}$ blood groups and infections from other Coronaviruses. For example, the study by Cheng et al. [5] reported that the SARS-CoV infection susceptibility in a group of health care workers in Hong Kong, exposed to an index SARS patient, was influenced by the ABO blood group systems; in particular, compared with non-O blood group hospital staff, blood group O hospital staff had a lower chance of getting infected. On a further investigation, Guillon et al. [10] found that either a 
monoclonal anti-A antibody or natural plasma anti-A specifically inhibited the SARS-CoV S protein/ACE2-dependent adhesion to ACE2-expressing cell lines. Therefore, ABO polymorphism could contribute to substantially reduce the SARS-CoV transmission, affecting both the number of infected individuals and the kinetics of the epidemic. A recent study by Lu et al. [11] has reported a structural similarity between the receptor-binding domains of SARS-CoV and SARSCoV-2; SARS-CoV and SARS-CoV-2 also use the same receptor, ACE2, for entry into target cells [12]. According to these findings, as suggested by Zhao et al., we can speculate that the lower susceptibility of blood group $\mathrm{O}$ and higher susceptibility of blood group A for COVID-19, as for SARS, could be linked to the presence of natural anti-blood group antibodies, particularly anti-A antibody, in the blood, which may inhibit the SARS-CoV-2 ACE2-dependent adhesion to target cells.

Based on the scientific evidence reported in this letter, we believe that further molecular, clinical and epidemiological studies are needed to investigate how the ABO polymorphism influences the host susceptibility, individual response and clinical risk for SARS-CoV-2 infection, but also whether it affects the kinetics of the COVID-19 pandemic, in order to promptly define adequate prevention and control strategies.

\section{Limitations}

This research note presents some limitations related to the previously published research we suggest to extend: Jiao Zhao et al. study presents some limitations, such as a limited population, scarce information on the control group, and scarce information on comorbidities. Furhermore, Zhao et al. meta-analysis is currently published as pre-print, and therefore has yet to be peer reviewed. Moreover, we are comparing the mechanisms that have been demonstrated in SARS$\mathrm{CoV}$ and those of SARS-CoV-2. However, more research is needed to give consistence to such assumption.

\section{List of abbreviations}

ACE2: Angiotensin converting enzyme 2; COVID-19: Coronavirus disease-2019; HBV: Hepatitis B virus; HIV: Human Immunodeficiency virus; OR: Odds Ratio; SARS: severe acute respiratory syndrome; WHO: World Health Organization.

\section{Declarations}

\section{Ethics approval and consent to participate}

Not applicable

Consent for publication

Not applicable

\section{Availability of data and materials}


Data sharing is not applicable to this article as no datasets were generated or analysed during the current study.

\section{Competing interests}

The authors declare that they have no competing interests.

\section{Funding}

The authors declare that they have not received any funding.

\section{Authors' contributions}

$D G$ and EB conceived the idea of the letter, reviewed the literature, and prepared the first draft. MPF reviewed and edited the final manuscript in collaboration with DG and EB for intellectual contents. All authors read and approved the final manuscript.

\section{Acknowledgments}

Not applicable

\section{Authors' information}

Not applicable

\section{References}

1. WHO - World Health Organization (2020). 'Situation report - 13, Novel Coronavirus (2019-nCoV), 2 February 2020'. https://www.who.int/emergencies/diseases/novel-coronavirus-2019/situation-reports

2. Chen N, Zhou M, Dong X, et al. Epidemiological and clinical characteristics of 99 cases of 2019 novel coronavirus pneumonia in Wuhan, China: a descriptive study. Lancet. 2020;395(10223):507-513. doi:10.1016/S0140-6736(20)30211-7.

3. Zhou F, Yu T, Du R, et al. Clinical course and risk factors for mortality of adult inpatients with COVID-19 in Wuhan, China: a retrospective cohort study [published correction appears in Lancet. 2020 Mar 28;395(10229):1038] [published correction appears in Lancet. 2020 Mar 28;395(10229):1038]. Lancet. 2020;395(10229):1054-1062. doi:10.1016/S0140-6736(20)30566-3.

4. Helmbold W, Vogel F. Correlations between $A B O$ blood groups and epidemic diseases and their anthropological significance. Bibl Haematol. 1962;13:279-280. doi:10.1159/000426428.

5. Cheng $\mathrm{Y}$, Cheng $\mathrm{G}$, Chui $\mathrm{CH}$, et al. $\mathrm{ABO}$ blood group and susceptibility to severe acute respiratory syndrome [published correction appears in JAMA. 2005 Aug 17;294(7):794. Cheng, Yufeng [corrected to Cheng, Yunfeng]]. JAMA. 2005;293(12):1450-1451. doi:10.1001/jama.293.12.1450-c.

6. Cooling L. Blood Groups in Infection and Host Susceptibility. Clin Microbiol Rev. 2015;28(3):801-870. doi:10.1128/CMR.00109-14.

7. Jing W, Zhao S, Liu J, Liu M. ABO blood groups and hepatitis B virus infection: a systematic review and meta-analysis. BMJ Open. 2020;10(1):e034114. Published 2020 Feb 2. doi:10.1136/bmjopen-2019034114.

8. Zhao J, Yang $\mathrm{Y}$, Huang $\mathrm{H}$, et al. Relationship between the ABO Blood Group and the COVID-19 Susceptibility. medRxiv 2020.03.11.20031096. doi: 10.1101/2020.03.11.20031096.

9. Chen J, Fan H, Zhang L, et al. Retrospective Analysis of Clinical Features in 101 Death Cases with COVID-19. medRxiv 2020.03.09.20033068; doi: 10.1101/2020.03.09.20033068. 
10. Guillon $P$, Clément M, Sébille V, et al. Inhibition of the interaction between the SARS-CoV spike protein and its cellular receptor by anti-histo-blood group antibodies. Glycobiology. 2008;18(12):1085-1093. doi:10.1093/glycob/cwn093.

11. Lu R, Zhao X, Li J, et al. Genomic characterisation and epidemiology of 2019 novel coronavirus: implications for virus origins and receptor binding. Lancet. 2020;395(10224):565-574. doi:10.1016/S01406736(20)30251-8.

12. Hoffmann M, Kleine-Weber $\mathrm{H}$, Krüger N, et al. The novel coronavirus 2019 (2019-nCoV) uses the SARS-coronavirus receptor ACE2 and the cellular protease TMPRSS2 for entry into target cells. bioRxiv 2020.01.31.929042. doi: 10.1101/2020.01.31.929042. 